\title{
The Circular Birefringence-Insensitive FBG Sensor for Weak Pressure
}

\author{
Hui Peng, Bi-hua Zhou, Li-hua Shi, Cheng Gao
}

National Key Laboratory on Electromagnetic Environment Effects and Electro-optical Engineering, PLA University of Science and Technology, Nanjing, Jiangsu, China.

Email: njnice99@163.com

Received August 2013

\begin{abstract}
The influence of the circular birefringence on the measurement performance was analyzed based on the Polarization Properties of FBG in this paper. Due to the circular birefringence, the linear relationship between the max of $P D L$ and pressure has been broken down. To estimate the cross sensitivity, a new parameter named relative peak of $P D L\left(R P_{P D L}\right)$ is proposed. Under different circular birefringence, the same pressure sensitivity of sensor has been achieved. The theoretical analysis and experiment results prove that transverse strain sensor of FBG is insensitive to the circular birefringence by applying the $R P_{P D L}$. This research can be provided to useful and practical application.
\end{abstract}

Keywords: Circularly Birefringence; Polarization Propertied; FBG Weak Pressure Sensor; The Relative Peak of PDL $\left(R P_{P D L}\right)$

\section{Introduction}

For both telecommunications and sensing purposes, FBG thus becomes important to characterize the polarization properties of FBG and their dependence on the wavelength [1-3]. Furthermore, this study can lead to the development of a new demodulation technique for FBGbased sensors. Caucheteur et al. have used polarization dependent loss (PDL) for transverse strain measurements $[4,5]$. The polarization dependent properties are also used for the magnetic field sensor [6].

Either way, there exist cross-sensitivity problems. In wavelength detection, the centre wavelength will change not only with the strain, but also with the temperature [7]. Similarly, in polarization detection, the polarization properties are influenced by the linear and circular birefringence [8,9]. Hence, we must take various kinds of measures to compensate or distinguish the cross-sensitivity problems. A number of techniques addressing this issue, such as dual-wavelength superimposed grating, two FBGs in different diameter fiber, hybrid FBG/long period grating, superstructure FBG and Fabry-Perot cavity method, have been reported [10-12]. Those methods provided some approaches to distinguish cross-sensitivity effect, but most of them needed specific gratings and special technique.

In this paper, the influence of the circular birefringence on the measurement performance was analyzed based on the polarization properties of FBG. To estimate the cross sensitivity, a new parameter named relative peak of PDL $\left(R P_{P D L}\right)$ is proposed. The simulation and experiment results proved that the relative peak of PDL $\left(R P_{P D L}\right)$ could effectively reduce the influence of circular birefringence.

\section{Theoretical Models}

\subsection{Principle of Measurement}

The polarization property of FBG has been widely used in the field of measurement. Here, we shortly introduce the principle of measurement.

The applied force causes a birefringence $\Delta n$, which is defined as the difference in refractive index between two orthogonal polarization modes called $x$ and $y$ modes (or eigenmodes). Due to the $\Delta n$, the $x$ and $y$ modes undergo different couplings through the grating. The total transmitted signal is the combination of the $x$ and $y$ mode signals. If there is only the linear birefringence, the Jones vector associated to the FBG transmitted signal is given by [5]:

$$
\left(\begin{array}{c}
E_{t, x} \\
E_{t, y}
\end{array}\right)=\left(\begin{array}{cc}
t_{x} & 0 \\
0 & t_{y}
\end{array}\right)\left(\begin{array}{l}
E_{i, x} \\
E_{i, y}
\end{array}\right)=\left(\begin{array}{c}
t_{x} E_{i, x} \\
t_{y} E_{i, y}
\end{array}\right)
$$

Where $\left(E_{i, x}, E_{i, y}\right)^{T}$ is the Jones vector of the input signal and $t_{x(y)}$ denotes the transmission coefficient of $x(y)$ mode FBG [13].

Polarization Dependent Loss $(P D L)$ is defined as the 
maximum change in the transmitted power by the grating as the input state of polarization is varied over all polarization states.

In this paper, the input signal is the linear state at $\pi / 4$ between the $x(y)$ mode, $E_{i, x}=E_{i, y}$. In the case of Bragg gratings, it is easy to show that the $P D L$ for the transmitted signal is given by [17]:

$$
\operatorname{PDL}(\lambda)=\left|10 \log _{10} \frac{T_{x}(\lambda)}{T_{y}(\lambda)}\right|=\left|10 \log _{10} \frac{E_{t, x}^{2}(\lambda)}{E_{t, y}^{2}(\lambda)}\right|
$$

\subsection{The Effect of Linear Birefringence}

The study of Caucheteur et al. demonstrated that there is linear relationship between the peak of $P D L$ and pressure [5]. But in practice, the fiber has intrinsic circular birefringence in the manufacturing process. Moreover, the induced circular birefringence can be caused by the shape, twisting and axial magnetic field of the fiber materials. The effect of circular birefringence must be taken into account when researching the performance of FBG weak pressure sensor based on the polarization properties.

Due to the circular birefringence, the Equation (1) will be modified as $[18,19]$ :

$$
\begin{gathered}
\left(\begin{array}{c}
E_{t, x} \\
E_{t, y}
\end{array}\right)=\left(\begin{array}{cc}
A & B \\
-B & A^{*}
\end{array}\right)\left(\begin{array}{cc}
t_{x} & 0 \\
0 & t_{y}
\end{array}\right)\left(\begin{array}{c}
E_{i, x} \\
E_{i, y}
\end{array}\right)=\left(\begin{array}{cc}
A & B \\
-B & A^{*}
\end{array}\right)\left(\begin{array}{c}
t_{x} E_{i, x} \\
t_{y} E_{i, y}
\end{array}\right) \\
A=\cos \left(\frac{\Phi}{2}\right)+j \frac{\delta}{\Phi} \sin \left(-\frac{2}{2}\right) \\
B=\frac{2 \Omega \Phi}{\Phi} \sin \left(-\frac{1}{2}\right) \\
\Phi=\left[\delta^{2}+(2 \Omega)^{2}\right]^{1 / 2} \\
2 \Omega=\left(\beta_{R}-\beta_{L}\right) l \\
\delta=\left(\beta_{x}-\beta_{y}\right) l
\end{gathered}
$$

Where $\Phi, 2 \Omega$ and $\Delta$ are the phase shift of elliptically, circularly and linear polarized light, respectively. And we use the $L$ and $R$ subscripts to indentify the eigenmodes corresponding to the left and circularly polarized light.

According to the Equations (2) and (3), we will understand more clearly the effects of circular birefringence on the evolutions of $P D L$.

\section{Simulation Results}

We design a FBG with 1.455 of $n_{\text {eff, }} 535 \mathrm{~nm}$ of $\Lambda, 4 \mathrm{~mm}$ of $L$ and $1 \times 10^{-4}$ of $\Delta n$. According to the given data, the simulation results can be got to analyze the influence of circular birefringence on the performance of proposed FBG sensor.

For purposes of analysis, some abbreviations were de- fined, such as $B_{P D L}$ (the value of PDL that outside the FBG band), $P_{P D L}$ (the peak value of PDL).

According to the Equations (1) and (2), Figure 1 presents the PDL as a function of pressure. As expected, the increase of pressure leads to a general increase of PDL amplitudes. The $P_{P D L}$ increase linearity with the pressure without circularly birefringence, which is shown in Figure 2.

Considering the influence of circular birefringence, the evolutions of $P D L$ spectrums with pressure and circular birefringence are shown in the Figure 3. Due to the circular birefringence, the $B_{P D L}$ is no longer zero and changed non-linearly with the circularly birefringence. Hence, the $P_{P D L}$ does not increase linearly with the increasing of pressure, as shown in Figure 3. From Figure 3, it also can be seen that the $P_{P D L}$ is influenced by the combination of circular and linear birefringence.

Up until now, it can be seen clearly that the linear relationship [5] between $P_{P D L}$ and pressure was broken because of circular birefringence. In order to eliminate the effect, a new parameter named the relative peak of $P D L$ $\left(R P_{P D L}\right)$ is defined. The $R P_{P D L}$ refers to the difference between the $B_{P D L}$ and $P_{P D L}$. Without circular birefringence, the $R P_{P D L}$ and $P_{P D L}$ are the same.

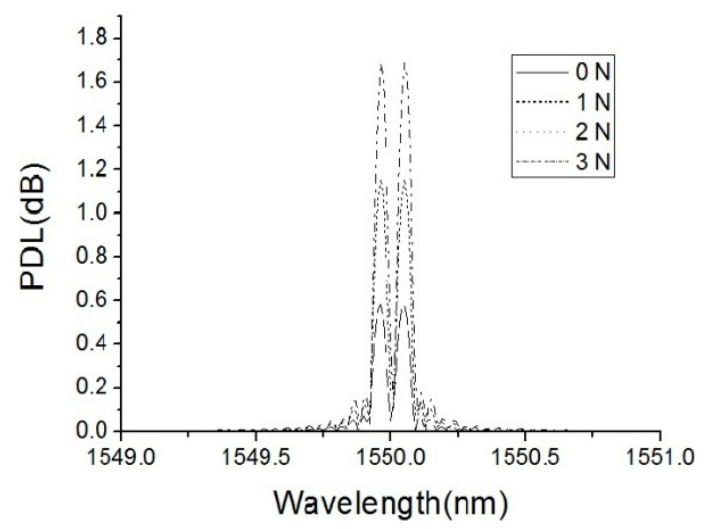

Figure 1. PDL versus wavelength at pressure without circularly birefringence.

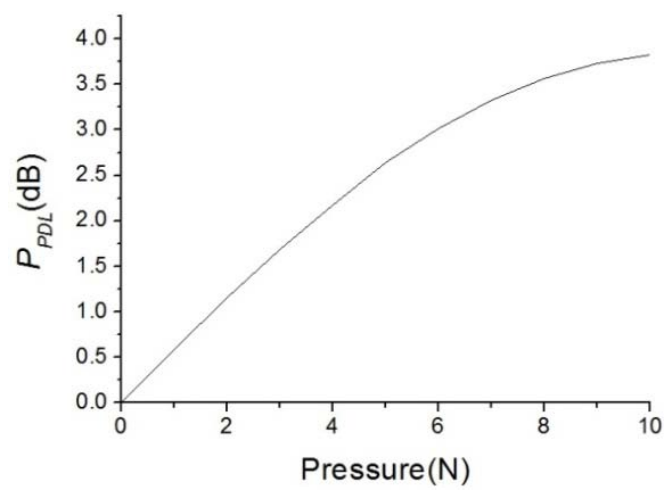

Figure 2. $P_{P D L}$ versus pressure without circularly birefringence. 


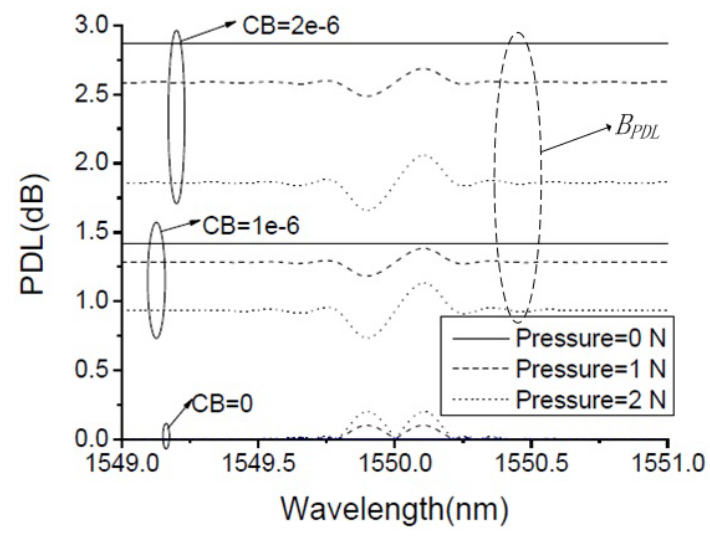

Figure 3. the evolutions of $P D L$ at different circularly birefringence and pressure ( $C B$ = circular birefringence).

Under circular birefringence, the evolutions of $R P_{P D L}$ were shown in Figure 4. From which it can be seen that the four $R P_{P D L}$ lines were almost coincided under difference circular birefringence (solid, dash, dot and dash dot). Figure 4 means that the $R P_{P D L}$ is insensitive to the circular birefringence. Hence, the influence of circular birefringence on the performances of the FBG pressure sensors can be eliminated by using the $R P_{P D L}$.

\section{Experiment Results}

The experimental data were then compared to theoretical evolutions. For that purpose, the experiment system was set up. The optical vector analyzer (OVA) is regarded as a light source, detector and processor. The FBG that used in experiment was designed and fabricated by our project group. The parameters of FBG are: $n_{\text {eff }}=1.455, \Lambda=535$ $\mathrm{nm}, \Delta n=5 \mathrm{e}^{-5}$ and $L=10 \mathrm{~mm}$. The width of glass plate is the same size as the length of FBG. The fixture was used to generate the random circular birefringence by twisted the FBG, in this way, only the qualitative method can be used to analyze the circular birefringence. Due to the experimental condition limitations, the applied pressure was in the range $1 \sim 10 \mathrm{~N}$ by steps of $1 \mathrm{~N}$. The results under two random conditions with different circular birefringence were calculated and analyzed in this section.

Using the optical vector analyzer whose precision is $10^{-5}(\mathrm{~dB})$ in our experiment [20], the $P D L$ evolutions for different pressure under two cases (different circular birefringence) are got and shown in Figure 5. The $P_{P D L}$ becomes more and more obvious along with the increase of pressure. To observe Figures $\mathbf{3}$ and $\mathbf{4}$, the simulation results and experiment results were similar.

Based on different pressure and circular birefringence, the experimental data of the $B_{P D L}, P_{P D L}$ and $R P_{P D L}$ values respectively are given in Table $\mathbf{1}$.

From the Figure 6, we can find that the two set of data about $R P_{P D L}$ and their fitting curves have a good agreement, which demonstrates that the $R P_{P D L}$ is not influ-

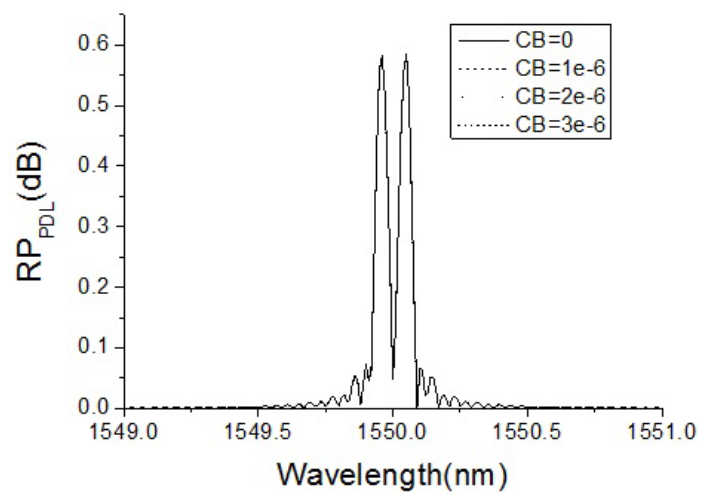

Figure 4. the evolutions of $R P_{P D L}$ at different circularly birefringence with same pressure $(C B=$ circular birefringence).

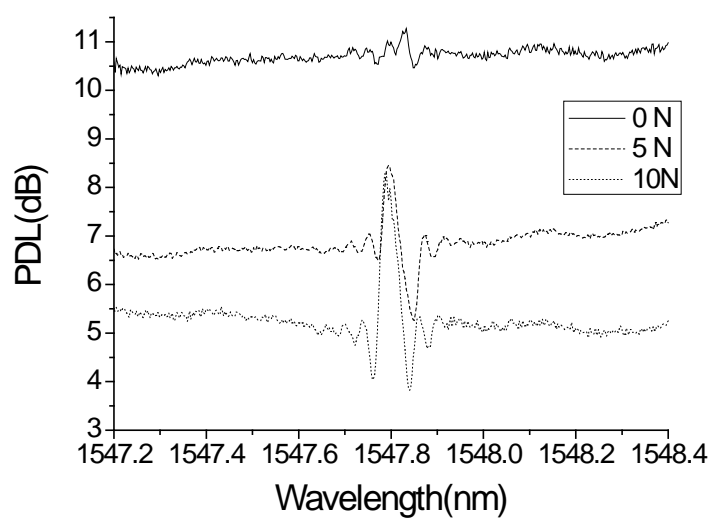

(a) case 1

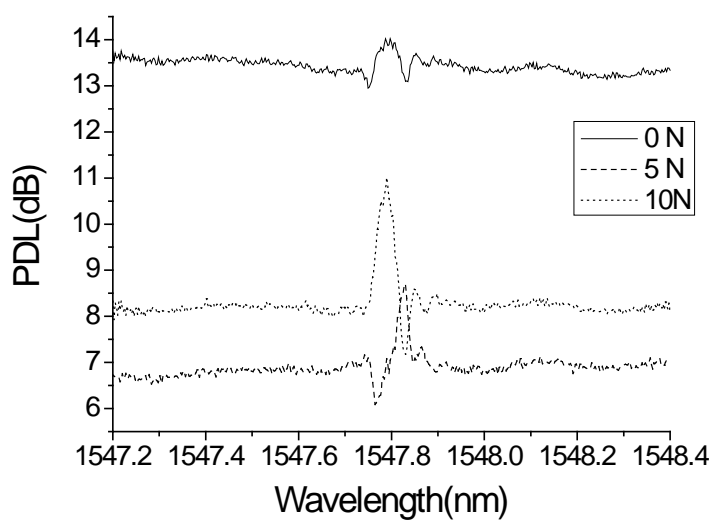

(b) case 2

Figure 5. PDL versus wavelength at pressure for two cases.

enced by the circular birefringence. In addition, due to the values of the fitting curves are increase monotonically with pressure, the $R P_{P D L}$ can be used to retrieve the pressure. Based on calculations, the pressure sensitivity are same $0.229 \mathrm{~dB} / \mathrm{N}$. Figure 6 also presents the theoretical results, the pressure sensitivity of which is 0.291 $\mathrm{dB} / \mathrm{N}$. The error between the experiment and theoretical results is caused by the manufacturing error of the FBG, such as the photo-induced birefringence [21,22]. The 
Table 1. the experimental results for two cases.

\begin{tabular}{|c|c|c|c|c|c|c|}
\hline \multirow{2}{*}{ Pressure/N } & \multicolumn{3}{|c|}{ Case 1} & \multicolumn{3}{|c|}{ Case 2} \\
\hline & $B_{P D L} / \mathrm{dB}$ & $P_{P D L} / \mathrm{dB}$ & $R P_{P D L} / \mathrm{dB}$ & $B_{P D L} / \mathrm{dB}$ & Peak/dB & $R P_{P D L} / \mathrm{dB}$ \\
\hline 0 & 10.94447 & 11.60567 & 0.6612 & 13.35158 & 14.01735 & 0.66577 \\
\hline 1 & 9.7146 & 10.36974 & 0.65514 & 11.3351 & 12.0727 & 0.7376 \\
\hline 2 & 6.94343 & 7.77878 & 0.83535 & 7.40321 & 8.23232 & 0.82911 \\
\hline 3 & 6.7089 & 8.0723 & 1.3634 & 7.5258 & 8.8028 & 1.277 \\
\hline 4 & 7.69596 & 9.12483 & 1.42887 & 8.1061 & 9.5944 & 1.4883 \\
\hline 5 & 5.79927 & 7.7797 & 1.98043 & 6.86557 & 8.69737 & 1.8618 \\
\hline 7 & 5.8658 & 7.91799 & 2.05219 & 6.98792 & 9.12278 & 2.13486 \\
\hline 8 & 6.37788 & 8.56389 & 2.18601 & 7.97609 & 10.19856 & 2.2225 \\
\hline 9 & 5.35373 & 7.74207 & 2.38834 & 6.08897 & 8.56583 & 2.47686 \\
\hline 10 & 6.3616 & 9.35989 & 2.99829 & 7.97609 & 10.95309 & 2.977 \\
\hline
\end{tabular}

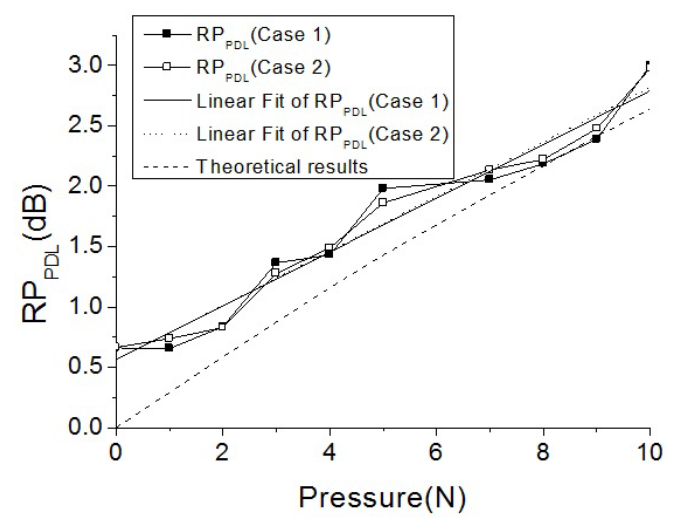

Figure 6. Evolution of $R P_{P D L}$ in response to a change of pressure.

initial value of $R P_{P D L}$ both are 0.66 under two conditions because of the intrinsic birefringence. The theoretical analysis and experiment results prove that the errors caused by the circular birefringence can be effectively eliminated by using the $R P_{P D L}$.

\section{Conclusion}

By analyzing the influences of the circular birefringence on the performance of the FBG pressure sensor, it is known that the nonlinear relationship between the $P_{P D L}$ and the pressure can be caused by the circular birefringence, which affects the accuracy of the FBG pressure sensor based on the $P_{P D L}$. For overcoming this weakness, the parameter denoted by $R P_{P D L}$ is proposed in this paper which can ensure the linear relationship between the $R P_{P D L}$ and the pressure under the different circular birefringence, thus the pressure can be retrieved by the values of the $R P_{P D L}$. The theoretical analysis and experiment results prove that the errors caused by the circular birefringence can be effectively eliminated by using the $R P_{P D L}$. This research is helpful to the practical application of the FBG weak pressure sensor, such as underwater acoustic and liquid level measurement, etc.

\section{Acknowledgements}

This work was supported by the National Natural Science Foundation of china under GRANT 61271106, the Jiangsu Province Natural Science Foundation BK2012508 and China Postdoctoral Science Foundation funded project (2012M521850). We would like to thank Prof. Xiangfei Chen of Nanjing University for providing OVA in experiment.

\section{REFERENCES}

[1] X. F. Yang, C. Y. Zhang, Z. R. Tong, et al., "Experimental Research of Temperature Sensing Properties of a Novel Fiber Grating,” Chinese Journal of Lasers, Vol. 38, No. 4, 2011, Article ID: 0405005.

[2] Y. Yu, Z. Meng and H. Luo, "Study of Fiber Bragg Grating Vibrating Sensors with Symmetry Push-Pull Configuration,” Semiconductor Optoelectronics, Vol. 32, No. 1, 2011, pp. 118-122.

[3] Y. N. Zhu, P. Shum, C. Lu, et al., "Temperature-Insensitive Fiber Bragg Grating Accelerometer,” IEEE PTL, Vol. 15, No. 10, 2003, pp. 1437-1439.

[4] C. Caucheteur, S. Bette, R. Garcia-Olcina, et al., "Transverse Force Sensor Exploiting the Birefringence Effect in Uniform Fibre Bragg Gratings," Proceedings of SPIE, Vol. 6585, 2007, 65850C/1-12 http://dx.doi.org/10.1117/12.722457

[5] C. Caucheteur, S. Bette and R. Garcia-Olcina, "Transverse Strain Measurements Using the Birefringence Effect in Fiber Bragg Grating," IEEE Photo Technology Letter, Vol. 19, No. 13, 2007, pp. 966-968.

[6] H. Peng, Y. Su and Y. Q. Li, "Analysis of a New Magnetic-Field Based on Polarized Parameters of Fiber Grating,” Journal of Microwaves, Vol. 25, No. 5, 2009, pp. 88-96.

[7] B. Zhang, G. S. Yan and Y. J. Deng, "Cross-Sensitivity of Fiber Grating Sensor Measurement," Journal of Applied Optics, Vol. 28, No. 5, 2007, pp. 614-618.

[8] F. Kui, Z. Yong, S. Yang and P. Hui, "Analysis of the Cross Sensitivity of Magnetic Field Sensor with Phase- 
Shift Grating,” Acta Optica Sinica, Vol. 30, No. 4, 2010, pp. 1020-1025.

[9] Y. Su, B. F. Zhang, Y. Zhu and Y. Q. Li, "Sensing Circular Birefringence by Polarization-Dependent Parameters in Fiber Bragg Gratings and the Influence of Linear Birefringence,” Opt. Fiber Technol, Vol. 18, 2012, pp. 51-57. http://dx.doi.org/10.1016/j.yofte.2011.11.007

[10] S. H. Aref, H. Latifi, M. I. Zibai and M. Afshari, "Fiber Optic Fabry-Perot Pressure Sensor with Low Sensitivity to Temperature Changes for Downhole Application,” Optics Communications, Vol. 269, No. 2, 2007, pp. 322-330.

[11] X. J. Ni, Y. Zhao and J. Yang, "Research of a Novel Fiber Bragg Grating Underwater Acoustic Sensor,” Sensors and Actuators A: Physical, Vol. 138, No. 1, 2007, pp. 76-80.

[12] Y. P. Wang, Y. J. Rao, Z. L. Ran, T. Zhu and X. K. Zeng, "Bend-Insensitive Long-Period Fiber Grating Sensors," Optics and Laser in Engineering, Vol. 41, No. 1, 2004, pp. 233-239.

[13] T. Erdogan, "Fiber Grating Spectra,” J. Llightwave Technol., Vol. 15, No. 8, 1997, pp. 1277-1294. http://dx.doi.org/10.1109/50.618322

[14] Z. Z. Li, "Theoretical and Experimental Research on Sensing Characteristics of Birefringenct Fiber Grating," National University of Defense Technology, 2006

[15] R. B. Wagreich, E. A. Altia, H. Singh and J. S. Sirkis, "Effects of Diametric Load on Fibre Bragg Gratings in Low Birefringence Fibre,” Electron. Lett., Vol. 32, No. 13, 1996, pp. 1223-1224. http://dx.doi.org/10.1049/el:19960806
[16] C. M. Lawrence, D. V. Nelson, E. Udd and T. Bennett, "A Fiber Optic Sensor for Transverse Strain Measurement,” Exp. Mech., Vol. 39, No. 3, 1999, pp. 202-209. http://dx.doi.org/10.1007/BF02323553

[17] S. Bette, C. Caucheteur, M. Wuilpart, et al., "Theoretical and Experimental Study of Differential Group Delay and Polarization Dependent Loss of Bragg Gratings Written in Birefringent Fiber," Optical Communication, Vol. 2. No. 269, 2007, pp. 331-337.

[18] Z. P. Wang, Q. B. Li, X. Y. Liu, et al., "Theoretical Analysis of the Effects of the Linear Birefringence upon the Performance of a Bulk Glass Optical Current Sensor," Acta Photonica Sinica, Vol. 33, No. 7, 2004, pp. 818-822.

[19] A. M. Smith, "Polarization and Magneto-Optic Properties of Single-Mode Optical Fiber,” Applied Optics, Vol. 17, No. 1, 1978, pp. 52-56.

[20] Optical Vector Analyzer User Guide, Document version 4. 7 for OVA control software version 3. 7. 1 m 2006 Luna Technologies, Inc

[21] H. Peng, "Research on Magnetic Field Measurement Based on Polarization Properties of Fiber Bragg Grating," Ph.D. Dissertation, PLA University of Science and Technology, Nanjing, 2009

[22] C. Caucheteur, S. Bette, F. Lhommé, et al., "Numerical Reconstruction of Induced Birefringence in Uniform Fiber Bragg Gratings Through Polarization Properties Measurement," Proceedings of 2005 IEEE/LEOS Workshop on Fibres and Optical Passive Components, 2005, pp. 228-233. 